\title{
Restoring riparian corridors with fire: Effects on soil and vegetation
}

\author{
ROBERT R. BLANK, JEANNE C. CHAMBERS, AND DESIDERIO ZAMUDIO
}

Authors are Soil Scientist, USDA Agricultural Research Service, Exotic and Invasive Weed Research Unit, 920 Valley Road, Reno, Nev. 89512, (blank@unr.nevada.edu), Research Ecologist, USDA Forest Service, Rocky Mountain Research Station, 920 Valley Road, Reno, Nev.89512; Soil Scientist, Fremont-Winema National Forest and the BLM Lakeview District, Lakeview Interagency Office, 1301 S. G Street, Lakeview Ore. 97630.

\section{Abstract}

In many riparian corridors of the semi-arid west, stream incision has resulted in lowered water tables, basin big sagebrush (Artemisia tridentata var. tridentata Nutt.) encroachment and the loss of the dominant herbaceous vegetation. To determine the potential for restoring basin big sagebrush-dominated riparian corridors to greater herbaceous cover, a fall prescribed burn on sites with relatively shallow $(-153$ to $-267 \mathrm{~cm})$ and deep $(-268$ to $\geq-300 \mathrm{~cm}$ ) water tables was conducted. We evaluated the separate and interacting effects of water table depth and burning on total soil $C$ and $N$, soil nutrient availability, and soil enzyme activities by microsite (sagebrush subcanopy, sagebrush interspace), and soil depth (ash/liter, 0-2, 2-5, and 10-20 cm). Three years after the prescribed burn, tissue nutrient content in silvery lupine (Lupinus argenteus Pursh) and Douglas sedge (Carex douglasii Boott), by microsite, growing in burned and unburned areas of 1 shallow water table site was measured. Influence of fire on soil attributes was largely limited to the top $5 \mathrm{~cm}$. As a consequence of prescribed burning, deep water table sites lost relatively more $\mathbf{N}$ and $\mathbf{C}$ from litter horizons and released more aqueous-soluble $\mathrm{Ca}^{+2}$ from $0-2 \mathrm{~cm}$ mineral horizons than did corresponding horizons from shallow water table sites. Overall, compared to unburned controls, burning: (1) increased aqueousextractable $\mathrm{SO}_{4}^{-2}, \mathrm{~K}^{+}$, and $\mathrm{KCl}$-extractable $\mathrm{NH}_{4}^{+}$, (2) decreased activities of the enzymes asparaginase, urease and acid-phosphatase, and (3) decreased $\mathrm{KCl}$-extractable $\mathrm{NO}_{3}{ }^{-}$and aqueoussoluble ortho-P. Out of 16 measured soil attributes reported, 7 were influenced by a microsite main effect and/or interaction. New tissue of silvery lupine on burned plots had greater $N$, greater $\mathrm{Zn}$ and $\mathrm{Fe}$ (only on subcanopy microsites) and less $\mathrm{Ca}$ than plants on control plots; new tissue of Douglas sedge had greater $\mathrm{S}$ and less $\mathrm{Na}, \mathrm{P}$, and $\mathrm{Zn}$. The results indicate that burning alone is an appropriate restoration treatment for shallow water table sites because of minimal $\mathrm{C}$ and $\mathrm{N}$ loss and increased available nutrients for regrowth of understory herbaceous species. Deep water table sites require a burning prescription that minimizes fire severity because of higher potential $\mathrm{C}$ and $\mathrm{N}$ loss, and reseeding due to a lack of perennial understory herbaceous species and more xeric conditions.

Key Words: restoration, riparian ecosystems, water table depth

Authors thank Ms. Tye Morgan and Dr. Dave Martin for field and laboratory assistance and Mr. Michael Wright for collection of plant material.

Manuscript accepted 2 Aug. 02

\section{Resumen}

En muchos corredores ribereños del oeste semiárido la incisión de corrientes ha resultado en mantos freáticos mas bajos, la expansión de "Basin big sagebrush" (Artemisia tridentata var. tridentata Nutt.) y la perdida de vegetación herbácea dominante. Para determinar el potencial para restaurar los corredores ribereños dominados por "Basin big sagebrush con una mayor cobertura herbácea se llevo a cabo un fuego prescrito en otoño en sitios con mantos freáticos relativamente poco profundos $(-153$ to $-267 \mathrm{~cm})$ y mantos freáticos profundos $(-268$ to $\geq-300$ cm). Evaluamos los efectos separados e interactuantes de la profundidad del manto fréatico y la quema en el $\mathrm{C}$ y $\mathbf{N}$ total del suelo, la disponibilidad de nutrientes del suelo $\mathrm{y}$ las actividades enzimáticas del suelo por micrositio ("Sagebrush" subcopa, "Sagebrush" interespacio) y profundidad del suelo (ceniza /mantillo, 0-2, 2-5 y 10-20 cm). Tres años después del fuego prescrito se midió por micrositio, el contenido de nutrientes de los tejidos de "Silvery lupine" (Lupinus argenteus Pursh) y "Douglas sedge" (Carex douglasii Boott), creciendo en áreas quemadas y sin quemar de un sitio con manto fréatico somero. La influencia del fuego en los atributos del suelo se limito principalmente a los 5 cm superiores. Como consecuencia del fuego prescrito, los sitios con manto fréatico profundo perdieron relativamente más $\mathrm{N}$ y $\mathrm{C}$ de los horizontes con mantillo y liberaron mas $\mathrm{Ca}^{+2}$ soluble en agua de los horizontes minerales de $0-2 \mathrm{~cm}$ que los horizontes correspondientes en los sitios con manto fréatico poco profundo. En general, comparado con los controles sin quemar, la quema (1) incremento el $\mathrm{SO}_{4}^{-2}$ extractables en agua, $\mathrm{K}^{+}$y $\mathrm{KCl}$ extractable $\mathrm{NH}_{4}^{+}$, (2) Disminuyo la actividad de las enzimas asparaginasa, ureasa y ácido- fosfatasa y (3) disminuyo el KClextractable, $\mathrm{NO}_{3}^{-}$y los ortho-P soluble en agua. De los 16 atributos de suelo medidos, 7 fueron influenciados por un efecto principal de micrositio y/o interacción. El tejido nuevo de "Silvery lupine"'en las parcelas quemadas tuvo mayor contenido de $N, Z n$ y Fe (solo en los micrositios de abajo de la copa) y menos Ca que las plantas en las parcelas control; el nuevo tejido del "Douglas sedge"tuvo más $\mathrm{S}$ y menos $\mathrm{Na}, \mathrm{P}$ y $\mathrm{Zn}$. $\mathrm{Z}$. Los resultados indican que la quema sola es un tratamiento apropiado para restauración de sitios con agua fréatica somera debido a las perdidas mínimas de $\mathrm{C}$ y $\mathrm{N}$ y el aumento en la disponibilidad de nutrientes para el rebrote de especies herbáceas. Los sitios con manto fréatico profundo requieren de una prescripción de fuego que minimice la severidad del fuego por el alto potencial de perdida de $\mathbf{C}$ y $\mathbf{N}$ y la resiembra debido a la falta de especies herbáceas perennes y a condiciones mas xéricas. 
Streams in the central Great Basin are in an incisional phase (Miller et. al. 2001), that is being exacerbated by anthropogenic disturbance (Chambers et al. 1998). Stream incision often results in lowered water tables and encroachment and dominance of basin big sagebrush (Artemisia tridentata var. tridentata Nutt.) in riparian corridors, and the loss of grass and forb-dominated meadow ecosystems. For riparian meadow ecosystems in general, the hydrologic regime, as indicated by water table depth, determines both soil properties (Chambers et al. 1999) and riparian ecosystem characteristics (Castelli et al. 2000). Thus, the potential for restoring sagebrush-dominated ecosystems to grass and sedge meadows depends on whether or not an abiotic threshold defined by water table depth has been crossed (Chambers and Linnerooth 2001). The characteristics of the soils and their response to restoration treatments are critical determinants of the restoration potential.

A restoration experiment was conducted to determine the potential for restoring basin big sagebrush- dominated riparian corridors to dry meadows. Two ecological site types were identified that occur naturally in the drainages and that have similar soils and landforms to serve as models for the restoration effort. The ecological site types are: (1) the dry meadow ecological site type which is dominated by grasses and sedges and has relatively shallow water tables; and (2) the basin big sagebrush/basin wild rye (Leymus cinereus Scribner \& Merr.) type. This type is dominated by basin big sagebrush and basin wild rye with lesser amounts of dry meadow species and has relatively deep water tables (Weixelman et al. 1996). A restoration treatment was applied to sites with relatively shallow and deep water tables that included a prescribed burn to remove basin big sagebrush followed by seeding herbaceous species typical of both ecological site types.

In this component of the larger restoration study, we examined the effects of both water table depth and prescribed burning on the soil and plant nutrient status of basin big sagebrush-dominated riparian corridors. The effects of fire on soil nutrient status in sagebrush steppe ecosystems are generally known (Blank et al. 1994a), but fires's effects on riparian corridors or interactions with water table depth have yet to be addressed. Moreover, there is limited data on how plant nutrient content is influenced when growing in post-fire soil. Therefore, this study asks 3 questions: 1) Does soil nutrient status dif- fer between sites with different water tables or in response to burning? 2) Does microsite influence post-fire soil nutrient status and nutrient uptake? 3) Do plants acquire soil nutrients at different rates in burned and unburned plots?

\section{Materials and Methods}

\section{Study Sites}

In July 1996, we located 4 basin big sagebrush-dominated study sites along Willow $\left(39^{\circ} 33^{\prime} 12^{\prime \prime} \mathrm{N}, 116^{\circ} 59^{\prime} 30^{\prime \prime} \mathrm{W}\right)$, Ledbetter $\left(39^{\circ} 45^{\prime} 30^{\prime \prime} \mathrm{N}, 117^{\circ} 30^{\prime} \mathrm{W}\right)$, and Marysville $\left(39^{\circ} 02^{\prime} 30^{\prime \prime} \mathrm{N}, 117^{\circ} 24^{\prime} 27^{\prime \prime} \mathrm{W}\right)$ Creeks in the Toiyabe Mountain Range of central Nevada that represented progressive stages of modification of the dry meadow ecosystem type. Study sites were on drainageways and stream terraces at elevations ranging from 2,000 to $2,300 \mathrm{~m}$, and were characterized by incised stream reaches. The climate is semiarid, and on average $72 \%$ of the 30 to $40 \mathrm{~cm}$ of average annual precipitation falls from MidOctober through May. Average temperature ranges from $-4^{\circ} \mathrm{C}$ in January to $20^{\circ} \mathrm{C}$ in July. Soil parent material is Quaternary alluvium of mixed mineralogy. The Willow Creek drainage is derived from granite, chert, shale, quartzite, limy sandstone and siltstone, and limestone. The Marysville and Ledbetter drainages are derived from quartz latite with a lesser amount of andesite and basalt.

Site selection was based on presence of species typical of the dry meadow and basin big sagebrush/giant wild rye ecosystem types (Weixelman et al. 1996), and depths to water table determined from hand-augered wells. Two hydro-geomorphic positions were included. Two study sites were located on lower benches and were characterized by relatively shallow water tables (Willow wet and Ledbetter Creeks; -153 to $-267 \mathrm{~cm}$ below the surface). An additional 2 sites were located on higher benches and had deeper water tables (Willow dry and Marysville Creeks; -268 to $\geq-300 \mathrm{~cm}$ below the surface). Sites with shallow water tables had higher cover of perennial graminoids and forbs, lower densities and volumes of basin big sagebrush, and were more similar to the model dry meadow type than shallow water table sites (Linnerooth et al. 1998). Graminoid species which typify the sites included Nevada bluegrass (Poa secunda spp. juncifolia [Scribner] R. Soreng), mat muhly (Muhlenbergia richardsonis [Trin] Rydb.), creeping wild rye (Leymus triticoides [Buckley] Pilger), basin wild rye, and Douglas sedge (Carex douglassi Boott). Common forbs included lupines (Lupinus sp.) and asters (Aster sp.). The shrubs, basin big sagebrush and green rabbitbrush (Chrysothamnus viscidiflorus ssp. viscidiflorus [Hook.] Nutt.), occurred with high constancy indicating that the study sites were relatively drier than the model dry meadow ecosystem type.

Holocene pedogenesis of Quaternaryaged sediments under herbaceous vegetation has produced deep, highly organic and relatively undifferentiated profiles. The soils on all sites are classified as Pachic Haplocryolls with $<2$-mm textures of loam and silt loam. Willow Creek dry and the Marysville site occur on slightly higher benches and are characterized by deeper water tables, the soils have lightercolored mollic epipedons. Coarse fragment content for all soils lies between 5 and $50 \%$.

\section{Experimental Design and Data Collection}

A paired plot approach was used in which 1 plot at each of the 4 sites received the restoration treatment (prescribed burn and seeding), and the other plot served as a control. Burning was conducted on 19-21 Oct. 1996 to remove both woody and understory vegetation. Plots were between 740 and $900 \mathrm{~m}^{2}$ in size, and fencing was used to reduce grazing and trampling by livestock and wildlife. Soils were sampled from 29 Oct. to 4 Nov. 1996. Sampling included both subcanopy and interspace microsites because of the effects of sagebrush plants on soil characteristics in these ecosystems. At each site, soil samples $(n=4)$ were collected for all restoration treatment/microsite combinations, and included the surface litter for unburned plots, surface ash for burned plots, and mineral soil depths of 0-2 cm, 2-5 cm, and 10-20 cm (2 water tables x 2 replicate sites $\mathrm{x} 2$ burn treatments $\mathrm{x} 2$ microsites $\times 4$ depths $\times 4$ subsamples). Surface samples were obtained with a trowel; deeper samples with a $10 \mathrm{~cm}$ diameter soil auger. From the time of the prescribed burn to when soil sampling occurred, approximately $12 \mathrm{~cm}$ of snow fell on the Willow Creek shallow and deep water table sites. Cold temperatures, however, limited snow melt and only the ash/litter layers and the upper $2 \mathrm{~cm}$ of the mineral soil became wet.

Vegetation was sampled in late June 1999 for both treatment/microsite combinations on the Willow Creek site with the deep water table. Three replicate transects were established within both the burned 
and unburned plots, 6 or 7 sample points were randomly located along each transect $(\mathrm{n}=20)$, and sample quadrats $\left(0.25 \mathrm{~m}^{2}\right)$ were placed in both the subcanopy and interspace microsites closest to the random point ( 2 burn treatments $\mathrm{x} 2$ microsites $\mathrm{x} 3$ replicate transects $\mathrm{x} 6$ or 7 subsamples). All plant material was clipped at the soil surface and separated by species. The vegetation samples were dried at $60^{\circ} \mathrm{C}$ for 48 hours and weighed (biomass is expressed as $\mathrm{g} \mathrm{m}^{-2}$ ). Only silvery lupine (Lupinus argenteus [Pursh]) and Douglas sedge on the Willow Creek site with the deep water table had sufficient biomass within all treatment/microsite combinations for analyses. This experimental design limits inferences of the results only to this site (see Hurlbert 1984).

\section{Laboratory Methods}

Soil samples were stored in an ice cooler in the field and at $2{ }^{\circ} \mathrm{C}$ between all preparatory steps in the lab. All soil, ash, and litter material was passed through a 2$\mathrm{mm}$ sieve prior to analysis. Some samples were dried slightly to facilitate sieving. All analyses were done on the $<2-\mathrm{mm}$ fraction. The dichromate oxidation procedure with supplemental heating was used to quantify organic carbon (OC) (Nelson and Sommers 1982). Total $\mathrm{N}$ was assayed by the micro-Kjeldahl procedure (Isaac and Johnson 1976). Aqueous soil extracts were prepared by shaking 10 grams soil with $20 \mathrm{ml}$ of deionized water for 1 hour (high organic matter samples required more water). The samples were centrifuged at $9400 \mathrm{x} \mathrm{g}$ for 10 minutes. Chloride, $\mathrm{HPO}_{4}{ }^{-2}$, and $\mathrm{SO}_{4}{ }^{-2}$ were quantified by gradient elution ion chromatography. Ion chromatography was used to quantify $\mathrm{Na}^{+}, \mathrm{K}^{+}, \mathrm{Mg}^{+2}$ and $\mathrm{Ca}^{+2}$. Ammonium and $\mathrm{NO}_{3}-$ were extracted with $0.5 \mathrm{M} \mathrm{KCl}(10 \mathrm{~g}$ soil $30 \mathrm{ml}$ extractant with shaking for $30 \mathrm{~min}$ ). Ammonium was quantified using flow-injection membrane diffusion colorimetric methodology. Nitrate was quantified by ion chromatography with spectroscopic detection at 210 $\mathrm{nm}$. Standards were made up from reagent grade chemicals. Soil enzyme bioassays of asparaginase, urease, and acid-phosphatase followed standard procedures (Tabatabai 1994). All data were corrected to $105^{\circ} \mathrm{C}$ soil weight.

Individual vegetation samples were ground in a Udy Mill and homogenized. Dry ashing followed by addition of $\mathrm{HCl}$ and $\mathrm{HNO}_{3}$ was used to solubilize metals (Campbell and Plank 1998). Calcium, Mg, $\mathrm{Fe}, \mathrm{Cu}, \mathrm{Mn}$, and $\mathrm{Zn}$ were quantified by atomic absorption spectroscopy; $\mathrm{Na}$ and $\mathrm{K}$ were quantified by atomic emission spectroscopy; $\mathrm{P}$ was quantified colorimetrically using molybdenum-blue chemistry. Nitrogen in ground plant tissue was quantified by the micro-Kjeldahl method (Isaac and Johnson 1976). We used the method of Kowalenko and Van Laerhoven (1998) to solubilize total plant $S$ which was quantified using ion chromatography.

\section{Statistical Analyses}

The overall study design was a split, split, split plot that was completely randomized at the main plot level. Water table depth (shallow, deep) was the main plot factor, treatment (burned, unburned) was the split plot factor, microsite (subcanopy, interspace) was the split, split plot factor, and depth was treated as a split, split, split plot factor (ash/litter, 0-2 cm, $2-5 \mathrm{~cm}, 10-20 \mathrm{~cm}$ ). Sites within water tables were the main plot error term. For significant $F$ values in the ANOVA model, Duncan's tests, at the $\leq 0.05$ probability level of significance, were used to separate means (SAS 1996). All significant main effects and interactions were examined, but only significant treatment main effects or interactions are reported. Vegetation data were analyzed separately by species (silvery lupine and Douglas sedge) using a 2-way ANOVA. Factors were treatment and microsite (subcanopy, interspace). For significant $F$ values, treatment differences were discerned at the $\mathrm{P} \leq 0.05$ level using L.S. means (SAS 1996).

\section{Results and Discussion}

\section{Visible Effects of Prescribed Burning}

Following prescribed burning, the pattern of fire effects mirrored the heterogeneous distribution of plant materials. Shrub subcanopies were covered with 1 to $5 \mathrm{~cm}$ of grayish to whitish ash which suggests temperatures greater than $750^{\circ} \mathrm{C}$ at the soil surface with lethal penetration of heat through the surface soil seedbank (Bentley and Fenner 1958). Lighter fuel loads in interspaces contributed to a darker ash generally less than $1 \mathrm{~cm}$ thick, which suggests temperatures somewhat greater than $350^{\circ} \mathrm{C}$ at this soil surface with lethal levels of heat only reaching to approximately $2 \mathrm{~cm}$ (Bentley and Fenner 1958). There were no visible differences in surface fire effect patterns between shallow water table and deep water table sites, but shallow water table sites had lower soil temperatures than deep water table sites (Chambers and Linnerooth 2001).

\section{Influence of Water Table Depth}

The response of the shallow and deep water table sites to the burn was more a function of the vegetation composition and structure than of the hydrologic regime. Depth to water table was not a significant main effect for any of the measured attributes (Table 1). Shallow water table sites had significantly more organic carbon (OC) in undershrub microsites than interspace microsites following the burn (water table $\mathrm{x}$ microsite interaction; Table 1) (Table 2), whereas deep water table sites had no significant differences in OC content between microsites. The different response was the result of a greater proportional loss of $\mathrm{OC}$, due to burning, in subcanopy microsites of deep rather than shallow water table sites. Similarly, prescribed burning reduced total soil N, averaged over all soil depths, but significantly more $\mathrm{N}$ was lost on deep rather than shallow water table sites (water table $\mathrm{x}$ treatment interaction; Table 1) (Table 2). Because the shallow water table sites support greater herbaceous plant cover and biomass and are generally moister than the deep water table sites (Wright 2001, Chambers and Linnerooth 2001), we expected them to contain more total soil $\mathrm{C}$ and N. This was not the case. For unburned sites, there were no differences in soil $\mathrm{C}$ and $\mathrm{N}$ between deep and shallow water table sites for any depth. The relatively greater loss of $\mathrm{C}$ and $\mathrm{N}$ from subcanopy microsites due to burning on deep rather than shallow water table sites can be attributed to greater fuel loads of sagebrush on deep water table sites (Linnerooth et al. 1998).

In the 0-2 cm depth increment, burning released significantly more $\mathrm{Ca}^{+2}$ from deep rather than shallow water table sites (water table depth $\mathrm{x}$ treatment $\mathrm{x}$ soil depth interaction; Table 1) (Table 3). For shallow water table sites, burning released much higher levels of $\mathrm{Mg}^{+2}$ in interspace microsites than in corresponding deep water sites (water table depth $\mathrm{x}$ treatment $\mathrm{x}$ microsite $\mathrm{x}$ soil depth interaction; Table 1) (Table 3). Increases in aqueous-soluble cations in upper soil layers and ash are often reported following fires (Smith 1970, Marion et al. 1991, Blank and Zamudio 1998). Greater shrub fuel loads on deep water table sites may have fostered a proportionally greater increase in aqueous-soluble $\mathrm{Ca}^{+2}$ and $\mathrm{Mg}^{+2}$ due to burning than occurred on shallow water table sites. For the shallow water table sites, large increases in post-burn levels of $\mathrm{Mg}^{+2}$ in ash layers of interspace microsites are possibly due to transfer of ash from subcanopy microsites. 
Table 1. ANOVA results for measured soil attributes showing $f$-values and significance levels with an asterisk.

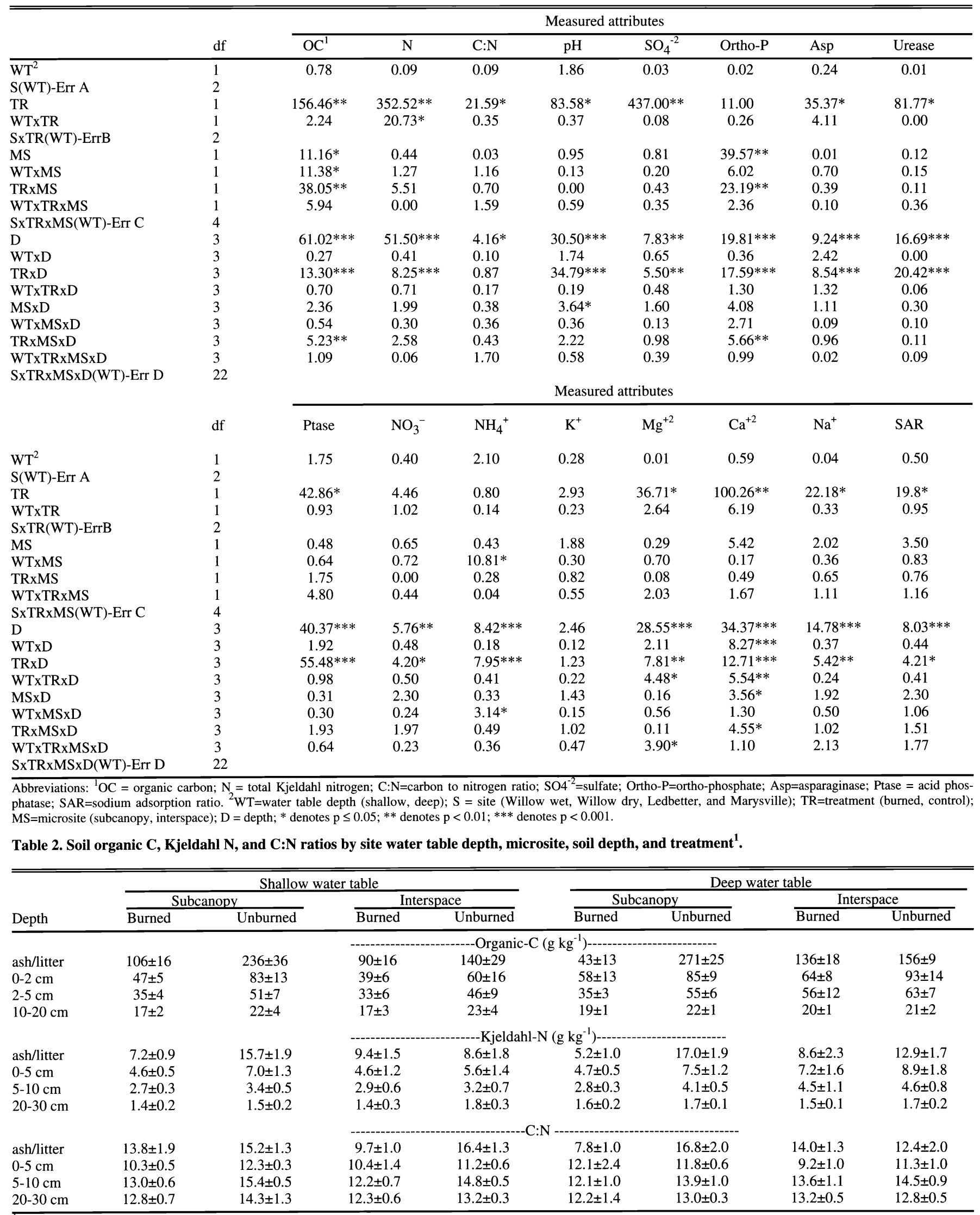

Variation in data shown by standard errors. 
Table 3. Aqueous-soluble cations and $\mathrm{KCl}$-extractable ammonium by site water table depth, microsite, soil depth, and treatment ${ }^{1}$.

\begin{tabular}{|c|c|c|c|c|c|c|c|c|}
\hline \multirow[b]{3}{*}{ Depth } & \multicolumn{4}{|c|}{ Shallow water table } & \multicolumn{4}{|c|}{ Deep water table } \\
\hline & \multicolumn{2}{|c|}{ Subcanopy } & \multicolumn{2}{|c|}{ Interspace } & \multicolumn{2}{|c|}{ Subcanopy } & \multicolumn{2}{|c|}{ Interspace } \\
\hline & Burned & Unburned & $\overline{\text { Burned }}$ & Unburned & Burned & Unburned & Burned & Unburned \\
\hline & & & ---------- & -Organic-C & aol $\left.\mathrm{kg}^{-1}\right)$ & ---------- & & \\
\hline ash/litter & $3.4 \pm 0.3$ & $4.3 \pm 0.7$ & $4.1 \pm 1.0$ & $2.2 \pm 0.8$ & $2.3 \pm 0.7$ & $3.2 \pm 0.5$ & $2.6 \pm 0.7$ & $3.2 \pm 0.8$ \\
\hline $0-2 \mathrm{~cm}$ & $2.9 \pm 1.0$ & $1.1 \pm 0.3$ & $1.3 \pm 0.4$ & $0.9 \pm 0.1$ & $7.4 \pm 1.6$ & $1.4 \pm 0.3$ & $3.1 \pm 0.9$ & $1.3 \pm 0.4$ \\
\hline $2-5 \mathrm{~cm}$ & $1.6 \pm 0.4$ & $0.8 \pm 0.1$ & $1.5 \pm 0.4$ & $0.5 \pm 0.1$ & $2.6 \pm 0.4$ & $1.6 \pm 0.7$ & $1.8 \pm 0.5$ & $1.2 \pm 0.2$ \\
\hline \multirow[t]{2}{*}{$10-20 \mathrm{~cm}$} & $0.6 \pm 0.1$ & $0.6 \pm 0.1$ & $0.5 \pm 0.2$ & $0.4 \pm 0.1$ & $0.5 \pm 0.1$ & $0.4 \pm 0.1$ & $0.6 \pm 0.2$ & $0.4 \pm 0.1$ \\
\hline & & & & $-\mathrm{Mg}^{+2}$ & $\left.\mathrm{~kg}^{-1}\right)$ & & & \\
\hline ash/litter & $8.0 \pm 1.9$ & $2.8 \pm 0.5$ & $13.0 \pm 5.0$ & $1.1 \pm 0.4$ & $7.7 \pm 1.1$ & $2.3 \pm 0.3$ & $2.7 \pm 1.4$ & $4.8 \pm 3.6$ \\
\hline $0-2 \mathrm{~cm}$ & $2.0 \pm 0.5$ & $0.7 \pm 0.1$ & $1.8 \pm 0.9$ & $0.5 \pm 0.1$ & $4.6 \pm 0.7$ & $1.0 \pm 0.2$ & $2.2 \pm 0.7$ & $0.7 \pm 0.1$ \\
\hline $2-5 \mathrm{~cm}$ & $1.2 \pm 0.3$ & $0.4 \pm 0.1$ & $1.0 \pm 0.3$ & $0.3 \pm 0.1$ & $2.3 \pm 0.5$ & $1.1 \pm 0.5$ & $1.4 \pm 0.6$ & $0.6 \pm 0.1$ \\
\hline \multirow[t]{2}{*}{$10-20 \mathrm{~cm}$} & $0.3 \pm 0.1$ & $0.2 \pm 0.1$ & $0.3 \pm 0.1$ & $0.2 \pm 0.1$ & $0.4 \pm 0.1$ & $0.3 \pm 0.1$ & $0.3 \pm 0.1$ & $0.2 \pm 0.1$ \\
\hline & & & & $\mathrm{Na}^{+}(\mathrm{r}$ & $\left.g^{-1}\right)---$ & ---------- & & \\
\hline ash/litter & $4.0 \pm 0.9$ & $1.8 \pm 0.5$ & $4.0 \pm 1.4$ & $0.8 \pm 0.2$ & $5.8 \pm 1.0$ & $0.9 \pm 0.2$ & $1.3 \pm 0.5$ & $1.3 \pm 0.8$ \\
\hline $0-2 \mathrm{~cm}$ & $1.3 \pm 0.3$ & $0.5 \pm 0.1$ & $0.7 \pm 0.2$ & $0.3 \pm 0.1$ & $2.2 \pm 0.5$ & $0.4 \pm 0.1$ & $1.5 \pm 0.4$ & $0.3 \pm 0.1$ \\
\hline $2-5 \mathrm{~cm}$ & $0.7 \pm 0.1$ & $0.4 \pm 0.1$ & $0.7 \pm 0.1$ & $0.3 \pm 0.1$ & $0.8 \pm 0.1$ & $0.5 \pm 0.2$ & $0.6 \pm 0.2$ & $0.3 \pm 0.1$ \\
\hline \multirow[t]{2}{*}{$10-20 \mathrm{~cm}$} & $0.4 \pm 0.1$ & $0.4 \pm 0.1$ & $0.4 \pm 0.1$ & $0.4 \pm 0.1$ & $0.5 \pm 0.1$ & $0.4 \pm 0.1$ & $0.3 \pm 0.1$ & $0.2 \pm 0.1$ \\
\hline & & & & $K^{+}(n$ & $\left.{ }^{-1}\right)$ & ---------- & & \\
\hline ash/litter & $112 \pm 38$ & $40.3 \pm 13.6$ & $46.4 \pm 8.0$ & $6.8 \pm 2.9$ & $241 \pm 95$ & $17.2 \pm 3.1$ & $8.1 \pm 2.9$ & $16.6 \pm 12.4$ \\
\hline $0-2 \mathrm{~cm}$ & $13.5 \pm 3.0$ & $7.0 \pm 1.7$ & $15.3 \pm 5.6$ & $2.2 \pm 0.5$ & $16.5 \pm 4.2$ & $9.1 \pm 3.4$ & $9.3 \pm 2.9$ & $1.7 \pm 0.4$ \\
\hline $2-5 \mathrm{~cm}$ & $10.1 \pm 1.9$ & $4.7 \pm 1.2$ & $13.7 \pm 5.2$ & $1.2 \pm 0.1$ & $14.4 \pm 1.8$ & $10.0 \pm 4.7$ & $9.7 \pm 4.9$ & $1.6 \pm 0.2$ \\
\hline \multirow[t]{2}{*}{$10-20 \mathrm{~cm}$} & $2.3 \pm 0.5$ & $2.2 \pm 0.7$ & $3.3 \pm 1.2$ & $1.9 \pm 0.6$ & $4.0 \pm 2.0$ & $2.7 \pm 0.8$ & $1.9 \pm 0.8$ & $1.0 \pm 0.2$ \\
\hline & & & & $-\mathrm{KC} 1-\mathrm{NH}$ & $\left.\mathrm{ol} \mathrm{kg}^{-1}\right)$ & & & \\
\hline ash/litter & $2.5 \pm 1.1$ & $10.9 \pm 8.4$ & $1.1 \pm 0.5$ & $4.6 \pm 1.9$ & $1.6 \pm 0.3$ & $3.8 \pm 1.0$ & $8.3 \pm 4.3$ & $10.4 \pm 1.8$ \\
\hline $0-2 \mathrm{~cm}$ & $8.4 \pm 2.1$ & $0.6 \pm 0.1$ & $3.6 \pm 0.9$ & $0.7 \pm 0.3$ & $7.5 \pm 0.8$ & $1.5 \pm 0.7$ & $7.1 \pm 1.2$ & $2.4 \pm 0.5$ \\
\hline $2-5 \mathrm{~cm}$ & $4.3 \pm 1.1$ & $0.7 \pm 0.3$ & $3.1 \pm 1.0$ & $0.3 \pm 0.1$ & $5.2 \pm 1.1$ & $1.3 \pm 0.7$ & $3.3 \pm 1.6$ & $0.9 \pm 0.3$ \\
\hline $10-20 \mathrm{~cm}$ & $0.3 \pm 0.1$ & $0.1 \pm 0.1$ & $0.6 \pm 0.3$ & $0.1 \pm 0.1$ & $0.6 \pm 0.2$ & $0.1 \pm 0.1$ & $0.5 \pm 0.3$ & $0.1 \pm 0.1$ \\
\hline
\end{tabular}

${ }^{1}$ Variation in data shown by standard errors.

In shallow water table sites, levels of $\mathrm{NH}_{4}{ }^{+}$tended to be lower with increasing soil depth for both microsites (water table $\mathrm{x}$ microsite $\mathrm{x}$ soil depth interaction; Table 1) (Table 3). For deep water table sites, levels of $\mathrm{NH}_{4}{ }^{+}$declined significantly with soil depth in interspace microsites, but were nearly constant for the first 3 depths in the subcanopy microsite and then declined significantly.

\section{Effects of Prescribed Burning on}

\section{Soil Attributes}

Significant loss of enzyme activity due to burning was largely limited to ash layers, but acid phosphatase activity was reduced to the $5 \mathrm{~cm}$ soil depth (urease, asparaginase and acid phosphatase all influenced by treatment $x$ depth interaction; Table 1) (Table 4). Although not significant, enzyme activity in burned subcanopies generally was lower compared to unburned controls. Soil enzyme activities declined due to heat deactivation (Saa et al. 1993, Hernandez et al. 1997, Staddon et al. 1998). The insulating properties of soil and brief exposure of the soil surface to high temperatures generally limits the depth of deactivation to $2 \mathrm{~cm}$ (Aston and Gill 1976). Greater loss of acid phosphatase activity deeper in the soil due to burning, relative to urease and asparaginase, may have occurred because acid

Table 4. Soil enzyme activities of urease, asparaginase, and acid phosphatase by site water table depth, microsite, soil depth, and treatment ${ }^{1}$.

\begin{tabular}{|c|c|c|c|c|c|c|c|c|}
\hline \multirow[b]{3}{*}{ Depth } & \multicolumn{4}{|c|}{ Shallow water table } & \multicolumn{4}{|c|}{ Deep water table } \\
\hline & \multicolumn{2}{|c|}{ Subcanopy } & \multicolumn{2}{|c|}{ Interspace } & \multicolumn{2}{|c|}{ Subcanopy } & \multicolumn{2}{|c|}{ Interspace } \\
\hline & Burned & Unburned & Burned & Unburned & Burned & Unburned & Burned & Unburned \\
\hline & & & 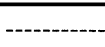 & ---Urease & $\left.\mathrm{hr}^{-1}\right)$ & ------------ & & \\
\hline ash/litter & $1.7 \pm 0.9$ & $36.4 \pm 7.6$ & $1.2 \pm 0.5$ & $45.8 \pm 23.3$ & $0.9 \pm 0.2$ & $41.5 \pm 6.9$ & $3.8 \pm 1.9$ & $34.6 \pm 7.8$ \\
\hline $0-2 \mathrm{~cm}$ & $2.6 \pm 1.3$ & $6.6 \pm 0.9$ & $4.9 \pm 1.1$ & $9.9 \pm 1.6$ & $1.3 \pm 0.4$ & $9.3 \pm 1.0$ & $3.9 \pm 1.1$ & $8.8 \pm 1.8$ \\
\hline $2-5 \mathrm{~cm}$ & $1.4 \pm 0.5$ & $3.5 \pm 0.7$ & $3.8 \pm 1.1$ & $8.2 \pm 0.9$ & $1.8 \pm 0.6$ & $4.4 \pm 0.6$ & $4.4 \pm 1.2$ & $5.3 \pm 1.1$ \\
\hline \multirow[t]{2}{*}{$20 \mathrm{~cm}$} & $5.0 \pm 0.8$ & $6.6 \pm 0.7$ & $4.0 \pm 0.9$ & $6.2 \pm 1.0$ & $4.2 \pm 0.7$ & $5.6 \pm 0.7$ & $4.6 \pm 0.7$ & $5.7 \pm 0.9$ \\
\hline & & & & -Asparagin & $\left.\mathrm{lg}^{-1} \mathrm{hr}^{-1}\right)$ & & & \\
\hline ash/litter & $0.9 \pm 0.4$ & $10.7 \pm 6.6$ & $0.6 \pm 0.2$ & $8.2 \pm 2.0$ & $0.8 \pm 0.1$ & $5.7 \pm 1.7$ & $1.0 \pm 0.5$ & $3.4 \pm 1.5$ \\
\hline $0-2 \mathrm{~cm}$ & $0.0 \pm 0.9$ & $1.0 \pm 0.1$ & $1.3 \pm 0.8$ & $0.8 \pm 0.2$ & $0.7 \pm 0.4$ & $1.1 \pm 0.2$ & $2.6 \pm 1.3$ & $1.6 \pm 0.5$ \\
\hline $2-5 \mathrm{~cm}$ & $0.8 \pm 0.2$ & $0.4 \pm 0.2$ & $0.0 \pm 0.3$ & $0.9 \pm 0.1$ & $0.7 \pm 0.3$ & $0.4 \pm 0.2$ & $0.7 \pm 0.3$ & $2.5 \pm 2.0$ \\
\hline \multirow[t]{2}{*}{$10-20 \mathrm{~cm}$} & $0.2 \pm 0.1$ & $0.7 \pm 0.3$ & $0.1 \pm 0.2$ & $0.8 \pm 0.2$ & $0.3 \pm 0.1$ & $0.3 \pm 0.1$ & $0.6 \pm 0.3$ & $0.7 \pm 0.2$ \\
\hline & & & & Icid Phosph & $\mathrm{mol} \mathrm{g}^{-1} \mathrm{hr}$ & & & \\
\hline ash/litter & $0.1 \pm 0.1$ & $9.0 \pm 1.4$ & $0.1 \pm 0.1$ & $10.4 \pm 3.3$ & $0.1 \pm 0.1$ & $13.3 \pm 1.0$ & $0.9 \pm 0.8$ & $11.7 \pm 1.1$ \\
\hline $0-2 \mathrm{~cm}$ & $1.0 \pm 0.3$ & $6.1 \pm 0.8$ & $2.0 \pm 0.8$ & $5.5 \pm 0.8$ & $0.7 \pm 0.3$ & $8.4 \pm 0.7$ & $2.2 \pm 0.7$ & $6.2 \pm 1.0$ \\
\hline $2-5 \mathrm{~cm}$ & $2.8 \pm 0.9$ & $4.2 \pm 0.4$ & $2.3 \pm 0.7$ & $5.8 \pm 1.0$ & $1.0 \pm 0.3$ & $6.0 \pm 0.8$ & $2.9 \pm 0.6$ & $4.9 \pm 0.7$ \\
\hline $10-20 \mathrm{~cm}$ & $1.6 \pm 0.2$ & $1.6 \pm 0.2$ & $1.3 \pm 0.2$ & $2.6 \pm 0.6$ & $1.1 \pm 0.1$ & $2.1 \pm 0.4$ & $1.3 \pm 0.2$ & $2.2 \pm 0.4$ \\
\hline
\end{tabular}

Variation in data shown by standard errors. 
Table 5. Soil pH and water-soluble sulfate and ortho-P and KCl-extractable nitrate by site water table depth, microsite, soil depth, and treatment ${ }^{1}$.

\begin{tabular}{|c|c|c|c|c|c|c|c|c|}
\hline \multirow[b]{3}{*}{ Depth } & \multicolumn{4}{|c|}{ Shallow water table } & \multicolumn{4}{|c|}{ Deep water table } \\
\hline & \multicolumn{2}{|c|}{ Subcanopy } & \multicolumn{2}{|c|}{ Interspace } & \multicolumn{2}{|c|}{ Subcanopy } & \multicolumn{2}{|c|}{ Interspace } \\
\hline & Burned & Unburned & Burned & Unburned & Burned & Unburned & $\overline{\text { Burned }}$ & Unburned \\
\hline ash/litter & $8.6 \pm 0.2$ & $6.3 \pm 0.3$ & $8.8 \pm 0.3$ & $5.8 \pm 0.2$ & $9.2 \pm 0.3$ & $5.9 \pm 0.1$ & $79+04$ & $56+02$ \\
\hline $0-2 \mathrm{~cm}$ & $7.1 \pm 0.1$ & $5.9 \pm 0.1$ & $7.4 \pm 0.4$ & $5.8 \pm 0.2$ & $6.8 \pm 0.3$ & $5.4 \pm 0.1$ & $6.9 \pm 0.3$ & $5.3 \pm 0.2$ \\
\hline $\mathrm{cm}$ & $6.4 \pm 0.2$ & $6.0 \pm 0.2$ & $6.8 \pm 0.4$ & $5.7 \pm 0.1$ & $6.3 \pm 0.1$ & $5.5 \pm 0.1$ & $6.0 \pm 0.3$ & $5.1 \pm 0.1$ \\
\hline $10-20 \mathrm{~cm}$ & $6.7 \pm 0.1$ & $7.1 \pm 0.2$ & $6.6 \pm 0.3$ & $6.5 \pm 0.2$ & $6.9 \pm 0.2$ & $6.5 \pm 0.1$ & $6.4 \pm 0.2$ & $6.5 \pm 0.1$ \\
\hline & & & & --Sodium a & ion ratio --- & & & \\
\hline ash/litter & $1.12 \pm 0.19$ & $0.65 \pm 0.12$ & $0.94 \pm 0.17$ & $0.47 \pm 0.13$ & $1.83 \pm 0.29$ & $0.39 \pm 0.06$ & $0.54 \pm 0.15$ & $0.37 \pm 0.12$ \\
\hline $0-2 \mathrm{~cm}$ & $0.58 \pm 0.06$ & $0.41 \pm 0.06$ & $0.43 \pm 0.05$ & $0.28 \pm 0.03$ & $0.62 \pm 0.11$ & $0.28 \pm 0.05$ & $0.60 \pm 0.09$ & $0.24 \pm 0.03$ \\
\hline $2-5 \mathrm{~cm}$ & $0.46 \pm 0.04$ & $0.38 \pm 0.08$ & $0.45 \pm 0.05$ & $0.35 \pm 0.03$ & $0.39 \pm 0.02$ & $0.28 \pm 0.05$ & $0.36 \pm 0.04$ & $0.20 \pm 0.03$ \\
\hline $10-20 \mathrm{~cm}$ & $0.56 \pm 0.09$ & $0.51 \pm 0.05$ & $0.63 \pm 0.18$ & $0.53 \pm 0.17$ & $0.53 \pm 0.07$ & $0.44 \pm 0.07$ & $0.33 \pm 0.05$ & $0.33 \pm 0.06$ \\
\hline & & & & $\mathrm{SO}_{4}{ }^{-2}$ & $\left.1 \mathrm{~kg}^{-1}\right)$ & & & \\
\hline ash/litter & $17.40 \pm 5.01$ & $2.70 \pm 0.98$ & $14.96 \pm 5.17$ & $0.43 \pm 0.24$ & $17.32 \pm 3.26$ & $1.28 \pm 0.27$ & $5.02 \pm 1.24$ & $0.65 \pm 0.27$ \\
\hline $0-2 \mathrm{~cm}$ & $5.62 \pm 1.47$ & $0.28 \pm 0.06$ & $4.11 \pm 1.63$ & $0.15 \pm 0.03$ & $9.40 \pm 2.46$ & $0.48 \pm 0.22$ & $5.41 \pm 1.78$ & $0.27 \pm 0.08$ \\
\hline $2-5 \mathrm{~cm}$ & $2.73 \pm 0.86$ & $0.14 \pm 0.03$ & $3.65 \pm 1.30$ & $0.12 \pm 0.03$ & $4.17 \pm 0.94$ & $0.48 \pm 0.33$ & $3.84 \pm 1.74$ & $0.20 \pm 0.04$ \\
\hline $10-20 \mathrm{~cm}$ & $0.28 \pm 0.11$ & $0.35 \pm 0.28$ & $0.85 \pm 0.39$ & $0.13 \pm 0.07$ & $1.52 \pm 0.54$ & $0.17 \pm 0.08$ & $0.97 \pm 0.51$ & $0.28 \pm 0.16$ \\
\hline & & & & ORTHO & $\left.\mathrm{mol} \mathrm{kg}^{-1}\right)$ & & & \\
\hline ash/litter & $0.26 \pm 0.11$ & $2.77 \pm 0.77$ & $0.18 \pm 0.05$ & $0.65 \pm 0.26$ & $0.25 \pm 0.06$ & $1.64 \pm 0.55$ & $0.49 \pm 0.19$ & $1.10 \pm 0.45$ \\
\hline $0-2 \mathrm{~cm}$ & $0.31 \pm 0.04$ & $0.65 \pm 0.12$ & $0.22 \pm 0.06$ & $0.14 \pm 0.01$ & $0.31 \pm 0.07$ & $0.66 \pm 0.13$ & $0.21 \pm 0.04$ & $0.20 \pm 0.04$ \\
\hline $2-5 \mathrm{~cm}$ & $0.30 \pm 0.05$ & $0.59 \pm 0.09$ & $0.17 \pm 0.04$ & $0.12 \pm 0.01$ & $0.35 \pm 0.05$ & $0.60 \pm 0.14$ & $0.23 \pm 0.06$ & $0.23 \pm 0.05$ \\
\hline $10-20 \mathrm{~cm}$ & $0.26 \pm 0.04$ & $0.33 \pm 0.05$ & $0.15 \pm 0.04$ & $0.13 \pm 0.03$ & $0.18 \pm 0.04$ & $0.23 \pm 0.06$ & $0.09 \pm 0.02$ & $0.17 \pm 0.04$ \\
\hline & & & & $--\mathrm{KCl}-\mathrm{NO}$ & $\left.\mathrm{nol} \mathrm{kg} \mathrm{kg}^{-1}\right)$ & & & \\
\hline ash/litter & $0.13 \pm 0.04$ & $2.87 \pm 2.15$ & $0.10 \pm 0.05$ & $0.66 \pm 0.55$ & $0.05 \pm 0.01$ & $4.18 \pm 1.30$ & $0.53 \pm 0.28$ & $3.44 \pm 2.49$ \\
\hline $0-2 \mathrm{~cm}$ & $0.50 \pm 0.31$ & $0.54 \pm 0.13$ & $1.05 \pm 0.50$ & $1.34 \pm 0.27$ & $0.10 \pm 0.07$ & $0.53 \pm 0.14$ & $0.59 \pm 0.26$ & $3.33 \pm 1.53$ \\
\hline $2-5 \mathrm{~cm}$ & $0.29 \pm 0.22$ & $0.29 \pm 0.07$ & $0.37 \pm 0.15$ & $0.52 \pm 0.10$ & $0.04 \pm 0.01$ & $0.31 \pm 0.09$ & $0.23 \pm 0.07$ & $1.41 \pm 0.45$ \\
\hline $10-20 \mathrm{~cm}$ & $0.13 \pm 0.02$ & $0.09 \pm 0.01$ & $0.13 \pm 0.03$ & $0.23 \pm 0.15$ & $0.05 \pm 0.01$ & $0.19 \pm 0.07$ & $0.08 \pm 0.03$ & $0.16 \pm 0.03$ \\
\hline
\end{tabular}

${ }^{1}$ Variation in data shown by standard errors.

phosphatase is more sensitive to heat, or compound(s) created by the fire may be inhibitory to acid phosphatase activity. Reduced enzyme activities of urease, asparaginase, and acid phosphatase can potentially lower mineralization rates of $\mathrm{N}$ and $\mathrm{P}$, at least in ash layers. The large reduction of acid phosphatase enzyme activity in burned subcanopies suggests possible interactions with $\mathrm{P}$ nutrition for plant establishment and regrowth.

Burned plots had lower organic carbon (OC) in the ash/litter and 0-2 cm depths than controls, with the greatest loss of OC occurring in shrub subcanopy microsites (treatment $\mathrm{x}$ microsite $\mathrm{x}$ soil depth interaction; Table 1) (Table 2). Nitrogen content was lower for all depths on burned than control plots, but was significantly lower only in ash/litter layers (treatment $\mathrm{x}$ depth interaction; Table 1) (Table 2). Carbon to $\mathrm{N}$ ratios were lower on burned plots (Table 2). Vegetation combustion and concomitant soil heating often leads to system loss of $\mathrm{C}$ and $\mathrm{N}$ and the level of loss is a function of microsite fuel levels (Schnitzer and Hoffman 1964, Raison 1979, DeBell and Ralston 1970, White et al. 1973, Blank et al. 1994a, Johnson et al. 1998). A consequence of lowered C:N ratios following the burns may be greater $\mathrm{N}$ mineralization potential (Christensen
1973, Stock and Lewis 1986, Dyrness et al. 1989, Kutiel and Naveh 1987).

Aqueous-soluble $\mathrm{Na}^{+}$and $\mathrm{KCl}$ extractable $\mathrm{NH}_{4}{ }^{+}$exhibited a significant treatment $\mathrm{x}$ soil depth interaction (Table 1). Prescribed burned plots had greater content of $\mathrm{Na}^{+}$in the ash/litter, 0-2 cm, and $2-5 \mathrm{~cm}$ depths than control plots (Table 3). Content of KCl-extractable $\mathrm{NH}_{4}{ }^{+}$was lower in the ash layer and higher in the $0-2 \mathrm{~cm}$ and $2-5 \mathrm{~cm}$ depths of the burned than control plots (Table 3). Large quantities of $\mathrm{NH}_{4}{ }^{+}$can be liberated after burns (Raison 1979, Khanna and Raison 1986, Overby and Perry 1996) depending on soil burn temperature (Marion et al. 1991). Extremely high temperatures, as occurred in the ash layers, fostered a slight increase in ammonium or slight loss relative to unburned litter layers. Beneath the ash layers where soil temperature was lower, ammonium levels increased markedly. The elevated extractable ammonium values in ash and mineral layers in burned treatments relative to controls suggests enhanced $\mathrm{N}$ nutrition for plants. High data variability and a conservative statistical approach negates any post-fire inferences regarding aqueous-soluble $\mathrm{K}^{+}$. However, $\mathrm{K}^{+}$was consistently higher in burned than control plots for all depths except 10-20 cm (Table 3).
Soil $\mathrm{pH}$, sodium adsorption ratio (SAR), aqueous-soluble $\mathrm{SO}_{4}{ }^{-2}$, and $\mathrm{KCl}$ extractable $\mathrm{NO}_{3}{ }^{-}$exhibited significant treatment $\mathrm{x}$ depth interactions (Table 1). Burning resulted in an increase in soil $\mathrm{pH}$, $\mathrm{SAR}$, and $\mathrm{SO}_{4}{ }^{-2}$ in all depths except $10-20$ $\mathrm{cm}$ (Table 5). Elevated soil reaction often occurs after fire and has been attributed to hydrolyses of basic cations (Sampson 1944). The pH's attained in our study due to burning, at least in ash layers, would negatively influence nutrient availability (Marschner 1995). The increase in SAR is due to a greater proportional release of $\mathrm{Na}^{+}$than $\mathrm{Ca}^{+2}$ plus $\mathrm{Mg}^{+2}$ due to burning. The SARs are below threshold values of about 10 which could lead to soil dispersion (Bohn et al. 1979). However, prescribed fires undertaken in soils with higher initial SARs may result in soil dispersion. Of all attributes measured, aqueous soluble $\mathrm{SO}_{4}{ }^{-2}$ increased the most following the burn, and was over 10 times greater in ash and mineral layers to $5 \mathrm{~cm}$ in burned than control plots. Although $\mathrm{S}$ readily volatilizes from vegetation heated above $300^{\circ} \mathrm{C}$ (Tiedemann 1987), oxidation of S-containing functional groups, possibly aided by catalysis on soil mineral surfaces, results in elevated soil sulfate levels after fires (Blank et al. 1994a). The large increase of aqueous-soluble $\mathrm{SO}_{4}{ }^{-2}$ 
due to fire should favorably affect $S$ nutrition of plants as the macronutrient is assimilated dominantly by root uptake (Marschner 1995).

In subcanopy microsites, ash layers had less ortho-P than the litter layer (treatment $\mathrm{x}$ microsite $\mathrm{x}$ depth interaction; Table 1) (Table 5). Soil content of ortho-P following vegetation fires is difficult to predict due to its involvement in numerous sorption and precipitation reactions, and to the interaction of these reactions with factors such as soil type, burn temperature, and potential volatilization (Raison et al. 1985). A decline in labile pools of ortho-P following fire can be due to precipitation with $\mathrm{Fe}$, $\mathrm{Al}$, and Ca (Smith 1970).

Burning resulted in loss of $\mathrm{NO}_{3}{ }^{-}$relative to controls for the litter/ash and $0-2 \mathrm{~cm}$ depths (treatment $\mathrm{x}$ soil depth interaction; Table 1) (Table 5). Loss of nitrate in surface soil layers following wildfire is almost universally reported in the literature and likely results from conversion to nitrogen gas and/or gaseous oxides of nitrogen at temperatures greater than $200^{\circ}$ C (DeBell and Ralston 1970, White et al. 1973). There may not be much ecological significance to these $\mathrm{NO}_{3}{ }^{-}$losses in sagebrush communities where mineralization can rapidly replenish $\mathrm{NO}_{3}{ }^{-}$(Blank et al. 1994a).

\section{Biomass and Nutrient Content of Vegetation}

In 1999, three years after the prescribed burn, total above ground herbaceous biomass for both the shallow and deep water table sites averaged $1,243 \mathrm{~g} \mathrm{~m}^{-2}$ on the burned plots and $469 \mathrm{~g} \mathrm{~m}^{-2}$ on control plots (Wright 2001). At the Willow Creek site with the shallow water table, the nitrogen fixing legume, silvery lupine, had higher aboveground biomass on the burned than control plot (Table 6). Aboveground biomass of Douglas sedge was higher on burned interspaces, but lower on burned subcanopies than corresponding controls. Tissue nutrient content of the 2 species varied considerably by treatment and microsite (Table 6). Silvery lupine had higher tissue content of $\mathrm{N}$ and lower content of $\mathrm{Ca}$ on the burned than control plot. Also, tissue content of $\mathrm{Zn}$ and Fe was higher on subcanopies than interspaces of the burned plot. For Douglas sedge, only tissue $\mathrm{S}$ was higher on the burned than control plot. Tissue $\mathrm{Zn}$ and $\mathrm{K}$ of Douglas sedge were higher in plants growing on the control than burned plot, and tissue $P$ was higher in subcanopies than interspaces on the control.

The stimulation of plant growth following fires or "ash-bed effect" is attributed to increased nutrient availability and uptake by plants (Cromer 1967, Kutiel and Naveh 1987). Luxury consumption of nutrients after fires may promote growth and reproduction in low nutrient soils (Rundel and Parson 1980). The influence of the "ashbed effect" may be short-lived, lasting less than one year (Renbuss 1968, Griffen and Friedel 1984). This may explain why we did not see overwhelming evidence of elevated nutrient uptake in burned plots. In a pot study, Blank et al. (1994b) grew big sagebrush (Artemisia tridentata spp. Wyomingensis Beatle \& A. Young), cheatgrass (Bromus tectorum L.), bottlebrush squirreltail (Elymus elymoides (Raf.) Swezey), and Indian ricegrass (Achnatherum hymenoides (Roemer \& Schultes) Barkworth) in the surface $5 \mathrm{~cm}$ of burned and unburned sagebrush subcanopy soil. Bottlebrush squirreltail and cheatgrass produced significantly more aboveground biomass when grown in burned soil, yet aboveground tissue concentrations of $\mathrm{N}, \mathrm{K}, \mathrm{P}, \mathrm{Ca}$, and $\mathrm{Mg}$ ( $\mathrm{S}$ and micronutrients were not quantified in this study) did not differ significantly between treatments. Although higher tissue concentrations of $\mathrm{S}, \mathrm{Fe}$, and $\mathrm{Zn}$ suggest increased uptake due to elevated soil availability on the burned plot, other factors may contribute to the increased abundance of herbaceous vegetation. Removal of sagebrush can enhance water availability (Sturges 1983), and the burned plots had higher late season moisture availability than the control plots (Chambers and

Table 6. Biomass and elemental content of aboveground plant material of Silvery lupine and Douglas sedge, by microsite and treatment, for the Willow Wet study site 1 .

\begin{tabular}{|c|c|c|c|c|c|c|c|c|c|}
\hline \multirow[b]{2}{*}{ Attribute } & \multicolumn{3}{|c|}{ Subcanopy } & \multicolumn{3}{|c|}{ Interspace } & \multicolumn{3}{|c|}{ Microsites Pooled } \\
\hline & Burned & & Unburned & Burned & & Unburned & Burned & & Unburned \\
\hline \multicolumn{10}{|c|}{ Silvery lupine } \\
\hline 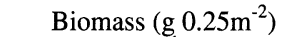 & $2.21 \pm 1.35$ & $*$ & $1.24 \pm 0.52$ & $9.64 \pm 3.98$ & $*$ & $2.67 \pm 1.34$ & $5.19 \pm 1.07$ & & $1.61 \pm 0.73$ \\
\hline $\mathrm{N}\left(\mathrm{mol} \mathrm{kg}^{-1}\right)$ & $2.6 \pm 0.1$ & & $1.9 \pm 0.1$ & $2.3 \pm 0.2$ & & $1.8 \pm 0.1$ & $2.4 \pm 0.1$ & $*$ & $1.9 \pm 0.1$ \\
\hline $\mathrm{Zn}\left(\mathrm{mmol} \mathrm{kg} \mathrm{kg}^{-1}\right)$ & $0.58 \pm 0.05$ & $*$ & $0.37 \pm 0.02$ & $0.39 \pm 0.03$ & $*$ & $0.50 \pm 0.03$ & $0.46 \pm 0.04$ & & $0.69 \pm 0.03$ \\
\hline $\operatorname{Mn}\left(\mathrm{mmol} \mathrm{kg}^{-1}\right)$ & $0.78 \pm 0.12$ & & $0.68 \pm 0.16$ & $0.52 \pm 0.04$ & & $0.71 \pm 0.15$ & $0.62 \pm 0.07$ & & $0.69 \pm 0.10$ \\
\hline $\mathrm{Fe}\left(\mathrm{mmol} \mathrm{kg}^{-1}\right)$ & $8.8 \pm 3.4$ & $*$ & $1.7 \pm 0.1$ & $2.7 \pm 0.9$ & & $4.3 \pm 1.1$ & $5.0 \pm 1.7$ & & $3.0 \pm 0.7$ \\
\hline $\mathrm{P}\left(\mathrm{mmol} \mathrm{kg}{ }^{-1}\right)$ & $74 \pm 6$ & & $72 \pm 1$ & $78 \pm 4$ & & $75 \pm 5$ & $76 \pm 3$ & & $73 \pm 3$ \\
\hline $\left.\mathrm{K}(\mathrm{mol} \mathrm{kg})^{-1}\right)$ & $0.38 \pm 0.03$ & & $0.45 \pm 0.03$ & $0.38 \pm 0.04$ & & $0.36 \pm 0.05$ & $0.38 \pm 0.03$ & & $0.41 \pm 0.03$ \\
\hline $\mathrm{Na}\left(\mathrm{mmol} \mathrm{kg}^{-1}\right)$ & $9 \pm 1$ & & $19 \pm 8$ & $17 \pm 7$ & & $33 \pm 10$ & $14 \pm 5$ & & $26 \pm 6$ \\
\hline $\mathrm{Ca}\left(\mathrm{mol} \mathrm{kg}{ }^{-\mathrm{P}}\right)$ & $0.22 \pm 0.01$ & & $0.30 \pm 0.02$ & $0.27 \pm 0.03$ & & $0.30 \pm 0.02$ & $0.25 \pm 0.02$ & $*$ & $0.30 \pm 0.01$ \\
\hline $\mathrm{S}\left(\mathrm{mmol} \mathrm{kg}^{-1}\right)$ & $39.0 \pm 13.7$ & & $35.9 \pm 0.7$ & $32.9 \pm 3.8$ & & $29.5 \pm 2.7$ & $35.2 \pm 2.6$ & & $32.7 \pm 1.7$ \\
\hline \multicolumn{10}{|c|}{ Douglas sedge } \\
\hline Biomass $\left(\mathrm{g} \mathrm{m}^{-2}\right)$ & $5.53 \pm 1.61$ & $*$ & $1.28 \pm 0.63$ & $0.49 \pm 2.84$ & $*$ & $4.81 \pm 1.76$ & $3.36 \pm 0.79$ & & $2.21 \pm 1.35$ \\
\hline $\mathrm{N}(\mathrm{mol} \mathrm{kg})$ & $1.1 \pm 0.1$ & & $1.0 \pm 0.1$ & $1.3 \pm 0.2$ & & $1.2 \pm 0.1$ & $1.2 \pm 0.1$ & & $1.2 \pm 0.1$ \\
\hline $\mathrm{Zn}\left(\mathrm{mmol} \mathrm{kg}{ }^{-1}\right)$ & $0.41 \pm 0.02$ & & $0.54 \pm 0.05$ & $0.35 \pm 0.02$ & & $0.50 \pm 0.05$ & $0.37 \pm 0.02$ & $*$ & $0.51 \pm 0.03$ \\
\hline $\mathrm{Mn}\left(\mathrm{mmol} \mathrm{kg}{ }^{-1}\right)$ & $0.58 \pm 0.06$ & & $0.52 \pm 0.09$ & $0.57 \pm 0.05$ & & $0.67 \pm 0.07$ & $0.57 \pm 0.04$ & & $0.62 \pm 0.06$ \\
\hline $\mathrm{Fe}\left(\mathrm{mmol} \mathrm{kg}^{-1}\right)$ & $5.7 \pm 1.0$ & & $4.6 \pm 1.0$ & $3.2 \pm 0.6$ & & $3.0 \pm 0.4$ & $4.3 \pm 0.7$ & & $3.5 \pm 0.5$ \\
\hline $\left.\mathrm{P}(\mathrm{mmol} \mathrm{kg})^{-1}\right)$ & $53 \pm 2$ & $*$ & $79 \pm 3$ & $55 \pm 4$ & & $59 \pm 1$ & $54 \pm 2$ & & $65 \pm 3$ \\
\hline $\mathrm{K}\left(\mathrm{mol} \mathrm{kg}^{-1}\right)$ & $0.37 \pm 0.02$ & & $0.49 \pm 0.02$ & $0.41 \pm 0.01$ & & $0.44 \pm 0.03$ & $0.39 \pm 0.01$ & $*$ & $0.46 \pm 0.02$ \\
\hline $\mathrm{Na}\left(\mathrm{mmol} \mathrm{kg}^{-1}\right)$ & $11 \pm 2$ & $*$ & $69 \pm 27$ & $13 \pm 2$ & & $24 \pm 11$ & $12 \pm 1$ & & $39 \pm 13$ \\
\hline $\mathrm{Ca}\left(\mathrm{mol} \mathrm{kg}{ }^{-1}\right)$ & $0.14 \pm 0.01$ & & $0.11 \pm 0.01$ & $0.13 \pm 0.01$ & & $0.14 \pm 0.01$ & $0.13 \pm 0.01$ & & $0.13 \pm 0.01$ \\
\hline $\mathrm{S}\left(\mathrm{mmol} \mathrm{kg}{ }^{-1}\right)$ & $49.2 \pm 4.3$ & & $49.2 \pm 1.3$ & $57.7 \pm 5.0$ & & $45.8 \pm 2.1$ & $53.9 \pm 3.5$ & $*$ & $46.4 \pm 1.5$ \\
\hline
\end{tabular}

An asterisk denotes significant differences due to treatment; significance judged at the $\mathrm{p} \leq 0.05$ level using Fisher's protected least significant difference. Variation in data shown by standard errors. 
Linnerooth 2001). Shading by sagebrush can reduce nutrient uptake by herbaceous vegetation (Cui and Caldwell 1997). Kutiel and Naveh (1987) report significant increase in shoot and root biomass and elemental content of $\mathrm{N}, \mathrm{P}, \mathrm{Mg}, \mathrm{K}, \mathrm{Ca}$, and $\mathrm{Fe}$ 2 months after fire for plants grown on ash.

\section{Conclusions and Implications}

This and companion studies show that while shallow water table sites can be restored to graminoid dominance with prescribed fire, deep water table sites appear to have crossed thresholds and will require both burning and reseeding. Three years after the burns, the shallow water table sites were dominated by species that characterize the dry meadow ecological site as a result of seedling establishment (Chambers and Linnerooth 2001) and especially vegetative growth and expansion of preexisting understory species (Wright 2001). In contrast, the deep water table sites were dominated by annual weedy species that occurred in the seed bank, including lambsquarter (Chenopodium album L.) and tansy mustard (Descurainia pinnata [Walt.] Britt.) (Wright 2001, Chambers and Wehking, unpublished data).

The soil response to the burns on the shallow vs deep water table sites was only indirectly related to the water table regime. There were no detectable differences between shallow and deep water table sites for the soil attributes measured. However, deep water table sites lost proportionately more $\mathrm{C}$ and $\mathrm{N}$ from subcanopy microsites, and released more aqueous soluble $\mathrm{Ca}^{+2}$ and $\mathrm{Mg}^{+2}$, than shallow water table sites. Also, higher mortality of both seeds and vegetative propagules occurred in subcanopy than interspace microsites, especially on deep water table sites (Wehking and Chambers, unpublished data). These differences can be attributed to higher fuel loads of basin big sagebrush (Linnerooth et al. 1998) and higher burn temperatures on deep rather than shallow water table sites. The water table regime influences the persistence and growth of basin big sagebrush in riparian corridors. Moreover, the biomass of basin big sagebrush, relative to understory herbaceous vegetation in degraded riparian corridors, can influence the effects of prescribed burns on both soils and vegetation. Prescribed burns on sites with high volumes of sagebrush and deep water tables can have more negative effects than on sites with lower volumes of sagebrush and shallower water tables.
The response of the soil chemical properties to prescribed burning were consistent with the existing literature, i.e., there is a general increase in water-soluble $\mathrm{Ca}^{+2}$, $\mathrm{Mg}^{+2}, \mathrm{Na}^{+}, \mathrm{SO}_{4}{ }^{-2}$, and $\mathrm{K}^{+}$, and $\mathrm{KCl}$ extractable $\mathrm{NH}_{4}^{+}$and a loss of extractable $\mathrm{NO}_{3}$. The increase in available nutrients undoubtedly influenced the response of both the perennial and herbaceous species following the burns. Even 3 years after burning, the content of selected nutrients in both silvery lupine and Douglas sedge was higher on burned than control plots on the deep water table site in Willow Creek. Our overall results indicate that prescribed burns on basin big sagebrush dominated sites with shallow water tables and a preexisting understory of dry meadow species result in minimal $\mathrm{C}$ and $\mathrm{N}$ loss and increased available nutrients for vegetative regrowth and seedling establishment. Burning sites with deep water tables, high sagebrush biomass, and few perennial herbaceous species can result in proportionately greater $\mathrm{C}$ and $\mathrm{N}$ loss, and increase available nutrients for weedy species. While burning alone is an appropriate restoration treatment for shallow water table sites, deep water table sites will require a burning prescription that minimizes fire severity, and reseeding with species adapted to more xeric conditions.

\section{Literature Cited}

Aston, A.R. and A.M. Gill. 1976. Coupled soil moisture, heat and water vapour transfers under simulated fire conditions. Aust. J. Soil Res. 14:55-66.

Bentley, J.R. and R.L. Fenner. 1958. Soil temperatures during burning related to postfire seedbeds on woodland range. J. For. 56:737-774.

Blank, R.R. and D.C. Zamudio. 1998. The influence of wildfire on aqueous-extractable soil solutes in forested and wet meadow ecosystems along the eastern front of the Sierra-Nevada Range, California. Intern. J. Wildl. Fire 8:79-85.

Blank, R.R., F. Allen, and J.A. Young. 1994a. Extractable anions in soils following wildfire in a sagebrush-grass community. Soil Sci. Soc. Am. J. 58:564-570.

Blank, R.R., F. Allen, and J.A. Young. 1994b. Growth and elemental content of several range species grown in unburned and post-wildfire soils and their influence on soil attributes. Plant Soil. 164:35-41.

Bohn, H.L., B.L. McNeal, and G.A. O'Connor. 1979. Soil chemistry. John Wiley \& Sons, New York, N.Y.

Campbell, C.R. and C.O. Plank. 1998. Preparation of plant tissue for laboratory analysis. p. 37-49. In: Y.P. Kalra (ed.) Handbook of reference methods for plant analysis. CRC Press, Boca Raton, Fla.

Castelli, R.M., J.C. Chambers, and R.J. Tausch. 2000. Soil-plant relations along a soil-water gradient in a Great Basin riparian meadow. Wetlands 20:251-266.

Chambers J.C. and A.R. Linnerooth. 2001. Restoring Artemisia dominated riparian corridors using alternative state and threshold concepts: Environmental and seedling establishment response. Appl. Veg. Sci. 4:157-166.

Chambers, J.C., R.R. Blank, D.C. Zamudio, and R.J. Tausch. 1999. Central Nevada riparian areas: Physical and chemical properties of meadow soils. J. Range Manage. 52:92-99.

Chambers, J.C., D. Farleigh, R.J. Tausch, J.R. Miller, D. Germanoski, D. Martin, and C. Nowak. 1998. Understanding longand short-term changes in vegetation and geomorphic processes: the key to riparian restoration. pp. 101-110. In: Potts (ed.) Rangeland Management and Water Resources. Amer. Water Resources Assoc. Herndon, Virg.

Christensen, N.L. 1973. Fire and the nitrogen cycle in California chaparral. Sci. 181:66-68.

Cromer, R.N. 1967. The significance of the "ashbed effect" in Pinus radiata plantations. Appita 20:104-112.

Cui, M. and M.M. Caldwell. 1997. Shading reduces exploitation of soil nitrate and phosphate by Agropyron desertorum and Artemisia tridentata from soils with patchy and uniform nutrient distributions. Oecologia. 109:177-183.

DeBell, D.S. and C.W. Ralston. 1970. Release of nitrogen by burning light forest fuels. Soil Sci. Soc. Amer. Proc. 34:936-938.

Dyrness, C.T., K. VanCleve, and J.D. Levison. 1989. The effect of wildfire on soil chemistry in four forest types in interior Alaska (USA). Can J. For. Res. 19:1389-1396.

Griffen, G.F. and M.H. Friedel. 1984. Effects of fire on central Australian rangelands. I Fire and fuel characteristics and changes in herbage and nutrients. Aust. J. Ecol. 9:381-393.

Hernández, T., C. García, and I. Reinhardt. 1997. Short-term effect of wildfire on the chemical, biochemical and microbiological properties of Mediterranean pine forest soil. Biol Fert. Soils 25:109-116.

Hurlbert, S.H. 1984. Pseudoreplication and the design of ecological field experiments. Ecol. Appl. 54:187-212.

Isaac, R. and W. Johnson. 1976. Determination of total nitrogen in plant tissue using a block digester. J. Assoc. Off. Anal. Chem. 59:98-100.

Johnson, D.W., R.B. Susfalk, R.A. Dahlgren, and J.M. Klopatek. 1998. Fire is more important than water for nitrogen fluxes in semi-arid forests. Environ. Sci. Policy 1:79-86.

Khanna, P.K. and R.F. Raison. 1986. Effect of fire intensity on solution chemistry of surface soil under a Eucalyptus pauciflora forest. Aust. J. Soil Sci. 24:423-434. 
Kowalenko, C.G. and Van Laerhoven, C.J. 1998. Total sulfur determination in plant tissue. p. 93-102. In: Y.P. Kalra (ed.) Handbook of reference methods for plant analysis. CRC Press, Boca Raton. Fla.

Kutiel, P. and Z. Naveh. 1987. The effect of fire on nutrients in a pine forest soil. Plant Soil 104:269-274.

Linnerooth, A.R., J.C. Chambers, and P.S. Mebine. 1998. Assessing the restoration potential of dry meadows using threshold and alternative states concepts. pp. 111-118. In: D.F. Potts (ed.) Rangeland Management and Water Resources. Amer. Water Resources Assoc. Herndon, Virg.

Marion, G.M., J.M. Moreno, and W.C. Oechel. 1991. Fire severity, ash deposition, and clipping effects on soil nutrients in Chaparral. Soil Sci. Soc. Amer. J. 55:235-240.

Marschner, H. 1995. Mineral nutrition of higher plants. Academic Press, San Diego. Calif.

Miller J., D. Germanoski, K. Waltman, R. Tausch, and J. Chambers. 2001. Influence of late Holocene processes and landforms on modern channel dynamics in upland watersheds of central Nevada. Geomorph. 38:373-391.

Nelson, D.W. and L.E. Sommers. 1982. Total carbon, organic carbon, and organic matter. p. 539-579. In: A.L. Page et al. (ed.) Methods of soil analysis part 2. $2^{\text {nd }} e d$. Agron. Monogr. 9. ASA and SSSA, Madison, Wisc.
Overby, S.T. and H.M. Perry. 1996. Direct effects of prescribed fire on available nitrogen and phosphorus in an Arizona chaparral watershed. Arid Soil Res. Rehab. 10:347-357.

Raison, R.J. 1979. Modification of the soil environment by vegetation fires, with particular reference to nitrogen transformations: A review. Plant and Soil. 51:73-108.

Raison, R.J., P.K. Khanna, and P.V. Woods. 1985. Mechanisms of element transfer to the atmosphere during vegetation fires. Can. J. For. Res. 15:132-139.

Renbuss, M.A. 1968. Microbial and nutrient changes associated with the "ashbed effect" M.Sc. Thesis. A.N.U. Canberra. Aust.

Rundel, P.W. and D.J. Parson. 1980. Nutrient changes in two chaparral shrubs along a fireinduced age gradient. Amer. J. Bot. 67:51-58.

Saa, A., M.S. Trasar-Cepeda, F. Gil-Sotres, and T. Caraballas. 1993. Changes in soil phosphorus and acid phosphatase activity immediately following forest fires. Soil Biol. Biochem. 25:1223-1230.

Sampson, A.W. 1944. Plant succession on burned chaparral lands in northern California. Univ. Calif. Agr. Expt. Sta. Bull. $685,144 \mathrm{p}$.

SAS Institute. 1996. SAS user's guide: statistics. SAS Inst., Cary, N.C.

Schnitzer, M. and I. Hoffman. 1964. Pyrolysis of soil organic matter. Soil Sci. Soc. Amer. Proc. 28:520-525.

Smith, D.W. 1970. Concentration of soil nutrients before and after fire. Can. J. Soil Sci. 50:17-29.
Staddon, W.J., L.C. Duchesne, and J.T. Trevors. 1998. Acid phosphatase, alkaline phosphatase and aryl-sulfatase activities in soil from a jack pine (Pinus banksiana Lamb.) ecosystem after clear-cutting, prescribed burning, and scarification. Biol. Fert. Soils 27:1-4.

Stock, W.D. and O.A.M. Lewis. 1986. Soil nitrogen and the role of fire as a mineralizing agent in a South African coastal fynbos ecosystem. J. Ecol. 74:317-328.

Sturges, D.L. 1983. Long-term effects of big sagebrush (Artemisia tridentata ssp. vaseyana) control on vegetation and soil water. J. Range Manage. 36:760-765.

Tabatabai, M.A. 1994. Soil enzymes. p. 775-833. In: Methods of Soil Analysis Part 2 Microbiological and Biochemical Properties Soil Sci. Amer. Madison, Wisc.

Tiedemann, A.R. 1987. Combustion losses of sulfur from forest foliage and litter. Forest Sci. 33:216-223.

Weixelman, D.A., D.C. Zamudio, and K.A. Zamudio. 1996. Central Nevada riparian field guide. USDA-For. Serv., Intermoun. Region R4-ECOL-96-01.

White, E.M., W.W. Thompson, and F.R. Gartner. 1973. Heat effects on nutrient release for soils under ponderosa pine. J. Range Manage. 26:22-24.

Wright, J.M. 2001. Restoring sagebrush-dominated riparian corridors using threshold and alternative state concepts: Aboveground vegetation response. Thesis, Univ. Nevada, Reno. 